\title{
Null Controllable Sets of Unstable Systems and their Application to MPC
}

\author{
Adrian Medioli*, Maria Seron*, Richard Middleton ${ }^{\dagger}$ \\ *The ARC Centre for Complex Dynamic Systems \& Control, The University of Newcastle, Australia \\ Email: Adrian.Medioli@newcastle.edu.au \\ $\dagger$ The Hamilton Institute, NUI Maynooth, Co Kildare, Ireland
}

\begin{abstract}
Model Predictive Control (MPC) is a popular advanced control strategy that is implemented by determining an optimal control sequence at each time step. One of the major contributors to MPC's computational complexity is the length of the control sequence or, equivalently, the number of optimisation parameters. This paper analyses the null controllable sets, and their related finite horizon approximation, of a class of unstable systems. The analysis reveals that the same control action can be obtained using a much smaller set of control moves via a technique which we term reduced parameterisation.
\end{abstract}

\section{INTRODUCTION}

This paper investigates the structure of the null controllable sets of a class of unstable systems with the aim of utilising this information in an MPC control strategy. More specifically the analysis investigates the controllable sets of input constrained open-loop unstable systems with an arbitrary number of real unstable modes. Previous work has analysed the structure and provided algorithms for generating approximations to the null controllable set (e.g., [1], [2], [3]). The analysis presented here extends the results to provide properties that can be applied in the context of MPC.

One technique for applying MPC with guaranteed stability to unstable systems, is to add the constraint that the unstable modes of the system are zero by the end of the finite control horizon $N$ [4]. This constraint can be re-stated in terms of $N$-step controllable sets. In essence they are the sets of initial states for which there exists an admissible control input sequence with the property that the states associated with the unstable modes are forced to the origin in $N$ moves. But the addition of this constraint, makes the controllable set dependent on the length of the control horizon and further reduces the region of attraction of the resulting closed-loop system. Enlarging the horizon to mitigate this problem necessarily increases the complexity of the associated optimisation problem. One of the key concepts explored in this paper for the reduction of MPC complexity is that of reduced parameterisation $^{1}$ (RP). RP is the process of describing a control sequence having $N$ control moves of equal duration via a sequence of $\mathrm{r}<N$, appropriately selected, control moves of (possibly) different duration. Indeed, since in the context of MPC the control moves are parameters of the associated optimisation problem, this RP substitution results

The authors gratefully acknowledge the support of Matrikon Asia-Pacific Pty Ltd, and the Australian Research Council.

${ }^{1}$ In the context of MPC this has also been called move blocking [5]. in a smaller number of parameters. Here, we investigate the effect that RP has on an $N$-step controllable set and develop the new concept of an RP controllable set. We show how the properties of RP controllable sets may be exploited in an MPC control strategy to reduce the complexity of the underlying optimisation problem.

\section{Class of Systems}

We consider a class of single input, open-loop unstable, linear systems described by the following discrete-time statespace model,

$$
x_{k+1}=A x_{k}+B u_{k},
$$

where $x_{k}=\left[\begin{array}{ll}x_{k \mid u}^{T} & x_{k \mid s}^{T}\end{array}\right]^{T}$,

$$
\begin{aligned}
A & =\left[\begin{array}{cc}
A_{u} & 0 \\
0 & A_{s}
\end{array}\right] ; B=\left[\begin{array}{l}
B_{u} \\
B_{s}
\end{array}\right], \\
u_{k} & \in \mathbb{U} \triangleq\left[u_{\min }, u_{\max }\right],
\end{aligned}
$$

and $A_{u}=\operatorname{diag}\left(a_{u 1}, \ldots, a_{u p}\right)$ is a diagonal matrix containing $p$ eigenvalues such that $a_{u p}>\ldots>a_{u 1}>1$, $A_{s}$ is a $q \times q$ matrix whose eigenvalues have modulus less than or equal to one, $B_{u}=\left[\begin{array}{lll}b_{u 1} & \ldots & b_{u p}\end{array}\right]^{T} \in \mathbb{R}^{p}$ and $B_{s}=\left[\begin{array}{lll}b_{s 1} & \ldots & b_{s q}\end{array}\right]^{T} \in \mathbb{R}^{q}$.

Further, $\left(A_{u}, B_{u}\right)$ is controllable and the input saturations values satisfy $u_{\min }<0$ and $u_{\max }>0$.

\section{Controllable Sets}

A controllable set is described as the set of all states that can be controlled to the origin with a control sequence that respects a given set of constraints.

\section{A. Null Controllable Set}

For input constrained unstable linear systems, the largest possible controllable set is the null controllable set $\mathcal{R}^{\max }$, defined as,

$$
\begin{aligned}
\mathcal{R}^{\max } \triangleq\left\{x_{0} \in \mathbb{R}^{n}: x_{k+1}=A x_{k}+B u_{k} ;\right. \\
\left.\lim _{k \rightarrow \infty} x_{k}=0 \text { and } u_{k} \in \mathbb{U} \text { for } k=0,1, \ldots\right\},
\end{aligned}
$$

where $A, B$ and $\mathbb{U}$ are as defined in (2)-(3).

Since the system is divided into unstable and stable (semistable) subsystems, $\mathcal{R}^{\max }$ can be divided into the two subsets $\mathcal{R}_{u}^{\max }$ and $\mathcal{R}_{s}^{\max }$ corresponding to the unstable and stable (semi-stable) null controllable sets of the system. That is, 
$\mathcal{R}^{\max } \triangleq \mathcal{R}_{u}^{\max } \times \mathcal{R}_{s}^{\max }$. Using the structure of the matrices in (2) we note that the unstable subset is given by

$$
\begin{array}{r}
\mathcal{R}_{u}^{\max }=\left\{x_{0 \mid u} \in \mathbb{R}^{p}: \lim _{k \rightarrow \infty} A_{u} x_{k \mid u}+B_{u} u_{k}=0\right. \\
\text { for } \left.u_{k} \in \mathbb{U}, k=0,1, \ldots\right\} .
\end{array}
$$

In addition, since the stable (semi-stable) states are not constrained, and [3, Proposition 1] has shown that $\mathcal{R}_{s}^{\max }=$ $\mathbb{R}^{q}$, the subsequent analysis does not consider $\mathcal{R}_{s}^{\max }$ unless specifically noted ${ }^{2}$.

\section{B. N-step Controllable Set}

For (1)-(2) the inputs are limited to the set $\mathbb{U}$ defined in (3). The addition of the constraint that the unstable states must be zero at the end of $N$ steps leads to what we have termed $N$-step controllable sets.

Using (1)-(2), the requirement that $x_{N \mid u}=0$ can be expanded as follows,

$$
x_{N \mid u}=A_{u}^{N} x_{0 \mid u}+\sum_{i=0}^{N-1} A_{u}^{i} B_{u} u_{N-1-i}=0 .
$$

Or equivalently, $x_{0 \mid u}=-\sum_{i=0}^{N-1} A_{u}^{-i-1} B_{u} u_{i}$. From this equivalent form of the constraints on the unstable states, we define $N$-step controllable sets.

Definition 1 ( $N$-step Controllable Set): The $N$-step controllable set for systems (1)-(3), denoted by $\mathcal{R}^{N}$, is defined as,

$$
\begin{aligned}
\mathcal{R}^{N}=\left\{x \in \mathbb{R}^{p}\right. & : x=-\sum_{i=0}^{N-1} A_{u}^{-i-1} B_{u} u_{i}, \\
& \text { for } \left.u_{i} \in \mathbb{U}, i=0, \ldots, N-1\right\},
\end{aligned}
$$

where $p$ is the number of unstable modes.

As an illustration of $N$-step controllable sets, Fig. 1a shows these sets for a system with two unstable modes for horizons of $N=5, N=20, N=50$, and the null controllable set.

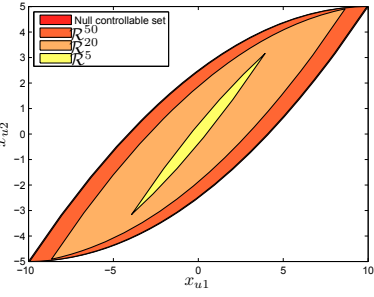

(a) Two unstable modes

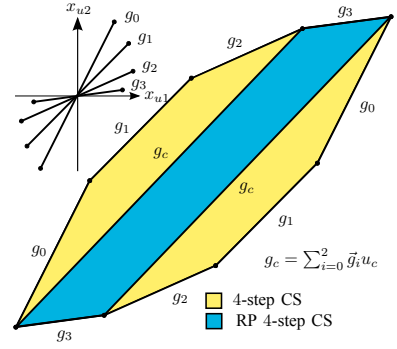

(b) Sets and Generators
Fig. 1: Sample $N$-step controllable sets.

We note that by taking $N$ large enough we obtain an $N$-step controllable set that is arbitrarily close to the null controllable set.

\section{Controllable Set Representations}

We will employ two alternative representations of the controllable sets, namely, the Minkowski sum representation and the $H$-polytope representation.

\footnotetext{
${ }^{2}$ For this reason in the sequel the $u$ subscript will be dropped from the state vector notation and $x$ will be inferred as representing the unstable subset of states unless otherwise noted.
}

1) Minkowski Sum Representation: From Definition 1 we note that $\mathcal{R}^{N}$ has an equivalent representation in terms of the Minkowski $\operatorname{sum}^{3}$ of a finite set of line segments, that is, $\mathcal{R}^{N}$ belongs to a class of polytopes called zonotopes [6]. Zonotopes are constructed by taking the Minkowski sum of a finite set of line segments called generators.

Definition 2 (Generator): A generator $g \subset \mathbb{R}^{p}$ is a line segment described in parametric form as

$$
g \triangleq\left\{x \in \mathbb{R}^{p}: x=\vec{g} \delta \text { for } \delta \in \mathbb{D}\right\},
$$

where $\vec{g}$ is the generator direction and $\mathbb{D}=\left[\delta_{\min }, \delta_{\max }\right]$ describes the range of the line segment.

More specifically for the zonotope $\mathcal{R}^{N}$, each of the generators used to construct $\mathcal{R}^{N}$ are given the term core generators.

Definition 3 (Core Generator): The $i^{\text {th }}$ core generator, $i=0,1, \ldots, N-1$, is the line segment

$$
g_{i} \triangleq\left\{x \in \mathbb{R}^{p}: x=\vec{g}_{i} u_{i} \text { for } u_{i} \in \mathbb{U}\right\}
$$

where the core generator direction $\vec{g}_{i}$ is given by

$$
\vec{g}_{i}=-A_{u}^{-i-1} B_{u}
$$

and $\mathbb{U}$ is as defined in (3).

Then, defining the set of core generators as

$$
\mathcal{G}^{N}=\left\{g_{0}, g_{1}, \ldots, g_{N-1}\right\},
$$

we have that $\mathrm{S}^{\oplus}\left(\mathcal{G}^{N}\right)=\mathcal{R}^{N}$. Fig. 1b illustrates 4 generators and their resulting 4-step controllable set.

Remark 1 (Generator Set Ordering): We note that the generator set $\mathcal{G}^{N}$ is ordered with respect to the sequential application of control inputs. This same ordering also describes the decreasing exponent of the $A_{u}$ matrix. Hence, throughout this paper, unless otherwise noted, generator sets are assumed to be ordered. The ordering property will extend to the set operations of union, intersection and difference, so that the resulting set is also ordered with respect to the sequential application of control inputs.

It will be useful for our analysis to study the facet structure of zonotopes. The facets of zonotopes are $(p-1)$-dimensional polytopes that have a close relationship to their generators. This relationship is detailed in the following theorem.

Theorem 1 (Facet Structure of Zonotopes): Let $\mathcal{R}^{d} \subset \mathbb{R}^{p}$ be a zonotope constructed by the Minkowski sum of generators $\mathcal{G}^{d} \triangleq\left\{g_{0}, g_{1}, \ldots, g_{d-1}\right\}, d \geq p$, where $g_{i}$, for $i=0, \ldots, d-1$, is defined in (7) with $\vec{g}=\vec{g}_{i}$ and where no two generators are collinear. Then any facet $\mathcal{F}_{i}^{d}$ has the following structure,

$$
\mathcal{F}_{i}^{d}=\mathrm{S}^{\oplus}\left(\mathcal{G}_{i}^{b, d}\right) \oplus z_{i}^{o, d} \text { for some } z_{i}^{o, d} \in \mathcal{Z}_{i}^{o, d}
$$

\footnotetext{
${ }^{3}$ The Minkowski sum of sets $\mathcal{A}=\left\{A_{1}, \ldots, A_{r}\right\}, A_{i} \subset \mathbb{R}^{p}$ for $i=1, \ldots, r$, denoted by $\mathrm{S}^{\oplus}(\mathcal{A})$, is defined as, $\mathrm{S}^{\oplus}(\mathcal{A}) \triangleq$ $\left\{x \in \mathbb{R}^{p}: x=\sum_{i=1}^{r} a_{i}\right.$, for $\left.a_{i} \in A_{i}, i=1, \ldots, r\right\}$. The Minkowski sum of a set $A_{1} \subset \mathbb{R}^{p}$ and a vector $a_{1} \in \mathbb{R}^{p}$ is denoted by $A_{1} \oplus a_{1}$.
} 
where $\mathcal{G}_{i}^{b, d} \subset \mathcal{G}^{d}$ is termed the "facet basis" generator set, $z^{o, d} \in \mathbb{R}^{p}$ is the "facet offset" vector and

$$
\begin{aligned}
& \mathcal{G}_{i}^{b, d}=\left\{g_{j}\right\}_{j \in \mathcal{I}_{i}^{b, d}}, \\
& \mathcal{Z}_{i}^{o, d}=\left\{z \in \mathbb{R}^{p}: z=\sum_{j \in \mathcal{I}_{i}^{o, d}} \vec{g}_{j} \delta_{j}^{s a t} \text { for } \delta_{j}^{s a t} \in \Delta\right\},
\end{aligned}
$$

and where $\mathcal{I}^{b, d}=\mathcal{I}^{d}$ choose $(p-1)=\left\{\mathcal{I}_{1}^{b, d}, \ldots, \mathcal{I}_{\mathrm{h}}^{b, d}\right\}$, $\mathcal{I}^{d}=\{0,1, \ldots, d-1\}, \mathcal{I}_{i}^{o, d}=\mathcal{I}^{d} \backslash \mathcal{I}_{i}^{b, d}, \mathrm{~h}=\left|\mathcal{I}^{b, d}\right|$. Further, the total number of facets is $2^{d-p+1} \mathrm{~h}$ and $\Delta=$ $\left\{\delta_{\min }, \delta_{\max }\right\}$.

Proof: See [7, Appendix B.1].

We note that Theorem 1 describes the general structure of facets, non-exposed or exposed, as consisting of a Minkowski sum of generators and an offset term. However, this theorem can not identify the single offset $z_{i}^{o, d}$ from the set $\mathcal{Z}_{i}^{o, d}$, that uniquely determines each facet of $\mathcal{R}^{d}$. In the sequel, we will be particularly interested in the exposed facets of $\mathcal{R}^{d}$, that is, those facets that constitute the polytope's "surface".

A fundamental concept in geometry is that all facets of a polytope, or in this case a zonotope, are supported by a hyperplane. Using this information, the following theorem shows how the offset $z_{i}^{o, d}$ that uniquely describes an exposed facet of $\mathcal{R}^{d}$ can be selected.

Theorem 2 (Exposed Facet Supporting Hyperplane):

Under the conditions of Theorem 1, the support hyperplane associated with an exposed facet $\mathcal{F}_{i}^{d}$ of $\mathcal{R}^{d}$ has the form

$$
\left\{x \in \mathbb{R}^{p}: \Gamma_{i} x=\gamma_{i}\right\},
$$

for some $i \in\{1, \ldots, \mathrm{f}\}$, where

$$
\Gamma_{i}=\left(\left\{\vec{g}_{j}\right\}_{j \in \mathcal{I}_{i}^{b, d}}\right)^{\perp},
$$

and where $\gamma_{i}$ takes one of the following forms:

$$
\gamma_{i}=\min _{z^{o, d} \in \mathcal{Z}_{i}^{o, d}} \Gamma_{i} z^{o, d} \text { or } \gamma_{i}=\max _{z^{o, d} \in \mathcal{Z}_{i}^{o, d}} \Gamma_{i} z^{o, d} .
$$

Proof: Let $\mathcal{F}_{i}^{d}$ be given as in (11). Then

$$
x \in \mathcal{F}_{i}^{d} \Longleftrightarrow x=\sum_{j \in \mathcal{I}_{i}^{b, d}} \vec{g}_{j} \delta_{j}+z_{i}^{o, d} \text { for some } \delta_{j} \in \mathbb{D} .
$$

It then follows that $x$ belongs to the hyperplane

$$
\left\{x \in \mathbb{R}^{p}: \Gamma_{i} x=\Gamma_{i} z_{i}^{o, d}\right\},
$$

since, by (15), $\Gamma_{i} \vec{g}_{j}=0$ for all $j \in \mathcal{I}_{i}^{b, d}$. Now, we note that (17) belongs to a family of parallel support hyperplanes associated with the normal $\Gamma_{i}$, each one produced by a selection of $z_{i}^{o, d} \in \mathcal{Z}_{i}^{o, d}$. Each exposed facet must be supported by one of the outermost hyperplanes of the form (17), which are given by (14), (16), proving the claim.

In the sequel the term facet will refer only to exposed facets.

\footnotetext{
${ }^{4}$ Given a set $\mathcal{V}$ and an integer $v, \mathcal{V}$ choose $v$ denotes the set of all unordered combinations of $v$ distinct elements from the set $\mathcal{V}$.

${ }^{5}(\mathcal{V})^{\perp}$, where $\mathcal{V}$ is a (set of) vectors, produces a vector that is orthogonal to all vectors in $\mathcal{V}$.
}

2) H-polytope Representation: Given the core generator set $\mathcal{G}^{N}$ defined in (10) and using Theorems 1, 2, we define the $H$-polytope representation ${ }^{6}$ of $\mathcal{R}^{N}$ as follows.

Definition 4 (H-polytope Representation of $\left.\mathcal{R}^{N}\right)$ : The $H$-polytope representation of $\mathcal{R}^{N}$ corresponding to the generator set $\mathcal{G}^{N}$ is given by,

$$
\mathcal{R}^{N} \triangleq\left\{x \in \mathbb{R}^{p}: \Gamma^{N} x \leq \gamma^{N}\right\},
$$

where $\Gamma^{N} \in \mathbb{R}^{\mathrm{f} \times p}$ is a row matrix, with each row $\Gamma_{i}^{N}$ as per (15) for $i=1, \ldots, \mathrm{f}$ and $\gamma^{N} \in \mathbb{R}^{\mathrm{f}}$ is a vector, with each row $\gamma_{i}^{N}$ for $i=1, \ldots, \mathrm{f}$, taking one of the forms (16), and where the different sets in Theorems 1 and 2 are constructed with $\mathcal{G}^{N}$ replacing $\mathcal{G}^{d}$.

\section{Reduced Parameterisation}

The action of standard MPC algorithms is to produce an optimal control sequence that satisfies all the imposed constraints. Given a horizon of length $N$ the algorithm produces a sequence of $N$ control inputs. When the initial state $x_{0}$ is on a vertex of the $N$-step controllable set $\mathcal{R}^{N}$, the only possible control sequence consists of saturated control inputs which occur in one or more groups of equal saturation values. This fact leads to the concept of RP, which is achieved by replacing each group of sequential control inputs that have the same saturation value with a single control input of a longer duration. For a selected RP a new controllable set $\mathcal{R}^{R P} \subset \mathcal{R}^{N}$ is produced, as explained below.

We first introduce the following definition.

Definition 5 (RP Sequence): An RP sequence $\mathcal{I}^{R P}$ is a set of sub-intervals from the interval $\mathcal{I}^{N} \triangleq\{0,1, \ldots, N-1\}$, defined as follows:

$$
\begin{aligned}
\mathcal{I}^{R P} \triangleq & \left\{\mathcal{I}_{i}^{R P} \subset \mathcal{I}^{N}: \mathcal{I}_{i}^{R P}=\left[s^{R P}(i), e^{R P}(i)\right],\right. \\
& s^{R P}(i), e^{R P}(i) \in \mathcal{I}^{N}, s^{R P}(i) \leq e^{R P}(i), \\
& \left.\bigcup_{i=0}^{\mathrm{r}-1} \mathcal{I}_{i}^{R P}=\mathcal{I}^{N}, \bigcap_{i=0}^{\mathrm{r}-1} \mathcal{I}_{i}^{R P}=\emptyset \text { for } i=0, \ldots, \mathrm{r}-1\right\},
\end{aligned}
$$

where $\mathrm{r} \leq N$ is the total number of parameters in the RP sequence. In addition the elements of $\mathcal{I}^{R P}$ are ordered by the relation $s^{R P}(i)<s^{R P}(i+1)$ for $i=0, \ldots, \mathrm{r}-2$. $\quad \circ$ The elements of each sub-interval of $\mathcal{I}^{R P}$ represent the indices of the control inputs that are equated to produce each new parameter in the RP. We next give the Minkowski sum and $H$-polytope representations of the RP sets $\mathcal{R}^{R P}$.

3) Minkowski Sum Representation: The effect of equating a group of sequential control inputs, is equivalent to replacing the associated group of generators with a single generator whose direction is the summation of directions of all generators from the group. The new replacement generator is termed a composite generator.

\footnotetext{
${ }^{6} \mathrm{An} H$-polytope $\mathcal{R}$ is a polytope described by the intersection of a finite set of closed half-spaces and is defined as, $\mathcal{R} \triangleq\left\{x \in \mathbb{R}^{n}: \Gamma x \leq \gamma\right\}$, where $\Gamma \in \mathbb{R}^{\mathrm{f} \times n}$ is a matrix whose rows contain support hyperplane "normals", $\gamma \in \mathbb{R}^{\mathrm{f} \times 1}$ is a vector of support hyperplane "offsets", $\mathrm{f}$ is the number of support hyperplanes and the inequality is interpreted element-wise. Further, unless otherwise noted each individual support hyperplane from the set is denoted by $\Gamma_{i} x=\gamma_{i}$ for $i=1, \ldots$, f.
} 
Definition 6 (Composite Generator): Given a sequential index set $\mathcal{I} \subset \mathcal{I}^{N}$ such that $|\mathcal{I}|>1$, the associated composite generator $g^{\mathcal{I}}$, with direction $\vec{g}^{\mathcal{I}} \triangleq \sum_{j \in \mathcal{I}} \vec{g}_{j}$, is

$$
g^{\mathcal{I}} \triangleq\left\{x \in \mathbb{R}^{p}: x=\vec{g}^{\mathcal{I}} u \text { for } u \in \mathbb{U}\right\} .
$$

Definition 7 (RP Generators): Given an RP index sequence $\mathcal{I}^{R P}=\left\{\mathcal{I}_{0}^{R P}, \ldots, \mathcal{I}_{\mathrm{r}-1}^{R P}\right\}$ as per Definition 5, the associated RP generators $g_{i}^{R P}, i=0, \ldots, \mathrm{r}-1$, with directions $\vec{g}_{i}^{R P} \triangleq \sum_{j \in \mathcal{I}_{i}^{R P}} \vec{g}_{j}$, are

$$
g_{i}^{R P} \triangleq\left\{x \in \mathbb{R}^{p}: x=\vec{g}_{i}^{R P} u_{i} \text { for } u_{i} \in \mathbb{U}\right\} .
$$

Therefore, the new set of generators that describe the RP set is given by the following definition.

Definition 8 (RP N-step Controllable Set): Given an RP index sequence $\mathcal{I}^{R P}$ as per Definition 5 and the associated RP generators $g_{i}^{R P}, i=0, \ldots, \mathrm{r}-1$, as per Definition 7, the resulting RP generator set $\mathcal{G}^{R P}$ is given by $\mathcal{G}^{R P}\left(\mathcal{I}^{R P}\right)=$ $\left\{g_{0}^{R P}, \ldots, g_{\mathrm{r}-1}^{R P}\right\}$, where $\mathrm{r}=\left|\mathcal{I}^{R P}\right|$.

Hence, the RP $N$-step controllable set $\mathcal{R}^{R P}$ is given by,

$$
\mathcal{R}^{R P}=\mathrm{S}^{\oplus}\left(\mathcal{G}^{R P}\left(\mathcal{I}^{R P}\right)\right) .
$$

Fig. 1b shows an example of an RP 4-step controllable that results from the use of a composite generator.

Remark 2 (RP Generator Set Ordering): The definition of RP generator sets produces a set that remains ordered with respect to the core generator set from which it is derived. That is, all composite generators appear in the set $\mathcal{G}^{R P}\left(\mathcal{I}^{R P}\right)$ at locations consistent with their interval of constituent core generators (as per Definition 5).

This is consistent with the requirement presented in Remark 1 for generator set ordering.

4) H-polytope Representation: The $H$-polytope representation of an RP $N$-step controllable set $\mathcal{R}^{R P}$ results from the same application of Theorems 1,2 , as that used in Definition 4 for the RP generator set $\mathcal{G}^{R P}\left(\mathcal{I}^{R P}\right)$.

Definition 9 (H-polytope Representation of $\left.\mathcal{R}^{R P}\right)$ : The $\mathrm{RP}$ set $\mathcal{R}^{R P}$, corresponding to an RP sequence $\mathcal{I}^{R P}$, results in the generator set $\mathcal{G}^{R P}$ which produces the $H$-polytope representation

$$
\mathcal{R}^{R P} \triangleq\left\{x \in \mathbb{R}^{p}: \Gamma^{R P} x \leq \gamma^{R P}\right\},
$$

where $\Gamma^{R P} \in \mathbb{R}^{\mathrm{f} \times p}$ and $\gamma^{R P} \in \mathbb{R}^{\mathrm{f}}$ are defined as in Definition 4 with $\mathcal{G}^{R P}$ replacing $\mathcal{G}^{N}$.

\section{A. Facet Structure of $\mathcal{R}^{N}$ and $\mathcal{R}^{R P}$}

This subsection uses the established representations and properties of both $\mathcal{R}^{N}$ and $\mathcal{R}^{R P}$ sets to describe the structure and properties of their facets.

Definition 10 (Facets of $\mathcal{R}^{N}$ ): For the $N$-step controllable set $\mathcal{R}^{N} \subset \mathbb{R}^{p}$ constructed from the Minkowski sum of generators $\mathcal{G}^{N} \triangleq\left\{g_{0}, \ldots, g_{N-1}\right\}$, the set of all facets is denoted by $\mathcal{F}^{N}$. Each facet of $\mathcal{F}^{N}$, denoted by $\mathcal{F}_{i}^{N}$ for $i=1, \ldots, \mathrm{f}$, is derived from the facet triple $\left(\mathcal{G}_{i}^{b, N}, \mathcal{G}_{i}^{o, N}\right.$, $\mathcal{U}_{i}^{o, N}$ ) as follows:

$$
\mathcal{F}_{i}^{N} \triangleq \mathrm{S}^{\oplus}\left(\mathcal{G}_{i}^{b, N}\right) \oplus z_{i}^{o, N},
$$

where $\mathcal{G}_{i}^{b, N}=\left\{g_{j}\right\}_{j \in \mathcal{I}_{i}^{b, N}}$ is a set of "facet basis" generators with indices in the set $\mathcal{I}_{i}^{b, N}$, defined as the $i^{\text {th }}$ element of $\mathcal{I}^{b, N}=\mathcal{I}^{N}$ choose $(p-1)$, with $\mathcal{I}^{N}=\{0, \ldots, N-1\}$. The facet offset $z_{i}^{o, N}$ is given by $z_{i}^{o, N}=\sum_{j \in \mathcal{I}_{i}^{o, N}} \vec{g}_{j} u_{i, j}^{o, N}$, where $\overrightarrow{\mathcal{G}}_{i}^{o, N}=\left\{\vec{g}_{j}\right\}_{j \in \mathcal{I}_{i}^{o, N}}$ is the set of "facet offset" generator directions, $\mathcal{I}_{i}^{o, N}=\mathcal{I}^{N} \backslash \mathcal{I}_{i}^{b, N}$, and $\mathcal{U}_{i}^{o, N} \triangleq\left\{u_{i, j}^{o, N}\right\}_{j \in \mathcal{I}_{i}^{o, N}}$ is the "facet offset" saturation set, where $u_{i, j}^{o, N} \in\left\{u_{\min }, u_{\text {max }}\right\}$ and the entire sequence is the optimising sequence of the facet's support hyperplane as per Theorem 2.

Definition 11 (Core Facet): A facet whose basis generator set includes only core generators as per Definition 3 is termed a "core facet".

It is of note that by this definition all facets of $\mathcal{R}^{N}$ are core facets.

Similarly, the facets of RP $N$-step controllable sets have the following structure.

Definition 12 (Facets of $\mathcal{R}^{R P}$ ): For the RP controllable set $\mathcal{R}^{R P} \subset \mathbb{R}^{p}$ constructed from the Minkowski sum of generators $\mathcal{G}^{R P} \triangleq\left\{g_{0}^{R P}, \ldots, g_{\mathrm{r}-1}^{R P}\right\}$, the set of all facets is denoted by $\mathcal{F}^{R P}$. Each facet of $\mathcal{F}^{R P}$ denoted by $\mathcal{F}_{i}^{R P}$ for $i=1, \ldots, \mathrm{f}$, is derived from the facet triple $\left(\mathcal{G}_{i}^{b, R P}, \mathcal{G}_{i}^{o, R P}\right.$, $\mathcal{U}_{i}^{o, R P}$ ) as follows:

$$
\mathcal{F}_{i}^{R P} \triangleq \mathrm{S}^{\oplus}\left(\mathcal{G}_{i}^{b, R P}\right) \oplus z_{i}^{o, R P},
$$

where $\mathcal{G}_{i}^{b, R P}$ and $z_{i}^{o, R P}$ are defined similarly to Definition 10 .

Definition 13 (Composite Facet): A facet whose basis generator set includes at least one composite generator as per Definition 6 is termed a "composite facet".

\section{B. Facet Structure Properties}

Having established representations and notation for the structure of facets, we next present results regarding the control sequences associated with trajectories originating on facets.

Lemma 1 (Control Sequence for $x_{0} \in \mathcal{F}_{i}^{N}$ ): Consider an initial state $x_{0}$ on the $i^{\text {th }}$ facet $\mathcal{F}_{i}^{N}$ of $\mathcal{R}^{N}$, where $x_{0}$ is expressed as (see Definition 10)

$$
x_{0}=\sum_{j \in \mathcal{I}_{i}^{b, N}} \vec{g}_{j} u_{j}^{*}+z_{i}^{o, N},
$$

with $u_{j}^{*} \in \mathbb{U}$ and $z_{i}^{o, N}=\sum_{j \in \mathcal{I}_{i}^{o, N}} \vec{g}_{j} u_{i, j}^{o, N}$.

Then an admissible control sequence that controls $x_{0}$ to the origin in $N$ steps is given by

$$
\left\{u_{0}, \ldots, u_{N-1}\right\}=\left\{u_{j}^{*}\right\}_{j \in \mathcal{I}_{i}^{b, N}} \bigcup\left\{u_{i, j}^{o, N}\right\}_{j \in \mathcal{I}_{i}^{o, N}} .
$$

Proof: Using (9), we can express $x_{0}$ as

$$
x_{0}=-\sum_{j \in \mathcal{I}_{i}^{b, N}} A_{u}^{-j-1} B_{u} u_{j}^{*}-\sum_{j \in \mathcal{I}_{i}^{o, N}} A_{u}^{-j-1} B_{u} u_{i, j}^{o, N} .
$$


Substituting (27) into $(5)^{7}$ gives

$$
\begin{aligned}
x_{N}= & -\sum_{j \in \mathcal{I}_{i}^{b, N}} A_{u}^{N-j-1} B_{u} u_{j}^{*}-\sum_{j \in \mathcal{I}_{i}^{o, N}} A_{u}^{N-j-1} B_{u} u_{i, j}^{o, N} \\
& +\sum_{k=0}^{N-1} A_{u}^{k} B_{u} u_{N-1-k} .
\end{aligned}
$$

Noting that $\sum_{k=0}^{N-1} A_{u}^{k} B_{u} u_{N-1-k}=\sum_{k=0}^{N-1} A_{u}^{N-k-1} B_{u} u_{k}$, and by using (26) results in $x_{N}=0$ as required.

The vertices of $\mathcal{R}^{N}$ describe the initial states that are only controllable via completely saturated control sequences. Also, by Lemma 1 we know that all points originating on a facet of $\mathcal{R}^{N}$ can be controlled by a combination of $(p-1)$ free control moves and $(N-p+1)$ saturated control moves.

From these observations, we conclude that RP is possible. However, RP requires the additional constraint that the sequence of control inputs must indicate the order in which the inputs are applied. Therefore only sequential inputs with equal saturation values may be grouped to produce a single control input with a longer duration. The following analysis uses the properties of the specified systems to describe which control inputs may be grouped.

We recall from Theorem 2, that each facet supporting hyperplane of $\mathcal{R}^{N}$ has an offset that is determined by an optimising control sequence. For the $i^{\text {th }}$ facet supporting hyperplane the offset is given by (see (16))

$$
\gamma_{i}^{N}=\Gamma_{i}^{N} \sum_{j=0}^{N-1} \vec{g}_{j} u_{i, j}^{s a t}
$$

where $\Gamma_{i}^{N}$ is the normal to the $i^{\text {th }}$ supporting hyperplane and $u_{i, j}^{\text {sat }} \in\left\{u_{\min }, u_{\max }\right\}$ for $j=0, \ldots, N-1$ is the sequence of controls that optimises $\gamma_{i}^{N}$. Hence, each $u_{i, j}^{s a t}$ is determined by the sign of $\Gamma_{i}^{N} \vec{g}_{j}$ for each $j \in\{0, \ldots, N-1\}$. Define $\Pi(j) \triangleq \Gamma_{i}^{N} \vec{g}_{j}$ and let $\Gamma_{i}^{N}=\left[\theta_{1}, \ldots, \theta_{p}\right]$. Using the definition of the generator directions (see (9)), and the structure of the matrices $A_{u}$ and $B_{u}$ defined in Section II, we can express $\Pi(j)$, for each $j \in\{0, \ldots, N-1\}$, as

$$
\Pi(j)=\sum_{k=1}^{p} \theta_{k} b_{u k} a_{u k}^{-j} .
$$

We now generalise the definition of $\Pi$ in (30) to be a function of a real variable, $\alpha \in \mathbb{R}$, as follows

$$
\Pi(\alpha)=\sum_{k=1}^{p} \theta_{k} b_{u k} a_{u k}^{-\alpha} .
$$

Note that by definition of the systems (1)-(3) all $a_{u k}$ are real and positive, therefore (31) is well defined and continuously differentiable. Hence, we have the following result.

Lemma 2 (Maximum Number of Zeros for $\Pi(\alpha)$ ): For any positive integer $p$, any set of distinct, positive $a_{u k}$, any set of $b_{u k} \neq 0$, for $k=1, \ldots, p$, and any non-zero vector $\Gamma_{i}^{N}=\left[\theta_{1}, \ldots, \theta_{p}\right] \in \mathbb{R}^{p}, \Pi(\alpha)$ has no more than $(p-1)$ real values of $\alpha$ for which $\Pi(\alpha)=0$.

Proof: See [7, Theorem 5.3].

Recalling that the sign of $\Pi(\alpha)$ for discrete values of $\alpha$ determines the optimising saturated control, we immediately have the following results.

\footnotetext{
${ }^{7}$ Letting $x_{0 \mid u}=x_{0}$ and dropping the $u$ subscript from $x_{N \mid u}$.
}

Theorem 3 (Feasible Sequence for Facets of $\mathcal{R}^{N}$ ): Consider the $i^{\text {th }}$ facet $\mathcal{F}_{i}^{N}$ of $\mathcal{R}^{N}$ and let $\mathcal{I}_{i}^{b, N}=$ $\{b(1), \ldots, b(p-1)\}$ represent the indices of the basis generators of the corresponding set $\mathcal{G}_{i}^{b, N}$.

Then any initial state $x_{0} \in \mathcal{F}_{i}^{N}$, can be controlled to the origin in $N$ steps by a feasible sequence $\mathcal{U}_{i}$ of the form

$$
\begin{aligned}
\mathcal{U}_{i} \triangleq & \left\{u_{[0, b(1)-1]}^{s a t}, u_{b(1)}, u_{[b(1)+1, b(2)-1]}^{s a t}, \ldots,\right. \\
& \left.u_{b(p-1)}, u_{[b(p-1)+1, N-1]}^{s a t}\right\}
\end{aligned}
$$

where $u_{b(1)}, \ldots, u_{b(p-1)}$ are "free" control moves that can take any value in the interval $\left[u_{\min }, u_{\max }\right]$ and $u_{[j, k]}^{s a t}$, for $j \leq k$, denotes a sequence of equal saturation values, that is,

$$
u_{[j, k]}^{s a t}=\left\{u_{j}^{s a t}, u_{j+1}^{s a t} \ldots, u_{k}^{s a t}\right\}
$$

where $u_{j}^{s a t}=u_{j+1}^{s a t}=\cdots=u_{k}^{s a t} \in\left\{u_{\min }, u_{\max }\right\}$ and $u_{[j, k]}^{s a t}=\{\}$ for $j>k$. Further, each sequence of equal saturation values has a value that is complementary (equal) to that of the previous sequence of equal saturation values if the number of free control moves separating them is odd (even).

Proof: Let $\mathcal{I}_{i}^{b, N}$ represent the set of indices of the generators from the basis set $\mathcal{G}_{i}^{b, N}$. By Theorem 2 the normal vector $\Gamma_{i}^{N}$ to a facet supporting hyperplane is constructed by taking the orthogonal complement of the generator directions from the basis set $\mathcal{G}_{i}^{b, N}$. This implies that for (31) we have,

$$
\Pi(\alpha)=0 \quad \text { for } \alpha \in \mathcal{I}_{i}^{b, N} .
$$

Since $\mathcal{I}_{i}^{b, N}$ has $p-1$ elements, then by Lemma 2 , the saturation value alternates at the indices corresponding to each basis generator but all saturation values in each interval between basis generators will be equal. Thus (32) is an admissible control sequence for states on the facet. The result then follows by Lemma 1.

Corollary 1 (Maximum Number of Reduced Parameters): At most there are $2 p-1$ parameters required to describe all feasible control sequences.

Proof: Allocating a parameter to each switch and interval identified in Theorem 3 proves the claim.

Corollary 2 (Feasible Sequence for Facets of $\mathcal{R}^{R P}$ ): Given an RP set $\mathcal{R}^{R P}$, all feasible control sequences for $x_{0} \in \mathcal{F}_{i}^{R P}$, where $\mathcal{F}_{i}^{R P}$ is the $i^{\text {th }}$ facet of $\mathcal{R}^{R P}$, satisfy the same sequencing property presented in Theorem 3 .

Proof: See [7, Corollary 5.3].

The following result shows that core facets of an RP set are also facets of its corresponding $\mathcal{R}^{N}$.

Lemma 3 (Core Facets of RP Sets): Let an $N$-step controllable set $\mathcal{R}^{N}$ be constructed from the sequence $\mathcal{G}^{N} \triangleq$ $\left\{g_{0}, \ldots, g_{N-1}\right\}$ of core generators with the index set $\mathcal{I}^{N}$. Let an RP $N$-step controllable set $\mathcal{R}^{R P}$ be constructed from an RP set $\mathcal{G}^{R P}$ resulting from an index sequence $\mathcal{I}^{R P}$ satisfying Definition 5 using $\mathcal{I}^{N}$. Then any core facet as per Definition 11 of $\mathcal{R}^{R P}$ is also a facet of $\mathcal{R}^{N}$.

Proof: We utilise properties of control sequences as per Theorem 3 to show that for a core facet $\mathcal{F}_{j}^{R P}$ (of the form (25)) there exists a facet of $\mathcal{F}_{i}^{N}$ (of the form (24)) such that $\mathcal{G}_{i}^{b, N}=\mathcal{G}_{j}^{b, R P}$ and $z_{i}^{o, N}=z_{j}^{o, R P}$ (see [7, Lemma 5.3]). 


\section{Application to MPC}

This section describes how the properties of $N$-step controllable sets and RP $N$-step controllable sets may be used in the formulation of MPC. The resulting RP problem is of reduced complexity and has a feasible set whose size is equal to that of MPC with $N$ optimisation parameters.

Firstly we present a definition describing the RP sequence with the smallest number of parameters that allows any state $x$ on a facet of $\mathcal{R}^{N}$ to be controlled to the origin in $N$ steps.

Definition 14 (RP Minimal Sequence): Let the facet $\mathcal{F}_{i}^{N}$ of the $N$-step controllable set $\mathcal{R}^{N}$, be given by (10). Let the indices of each generator in the facet basis set $\mathcal{G}_{i}^{b, N}$ be defined as the set $\mathcal{I}_{i}^{b, N} \triangleq\{b(1), \ldots, b(p-1)\}$ then the minimal RP sequence associated with $\mathcal{F}_{i}^{N}$, is given by the index set

$$
\begin{aligned}
\mathcal{I}_{i}^{\mathcal{F}_{i}^{N} \triangleq} & \{[0, b(1)-1], b(1),[b(1)+1, b(2)-1], b(2), \ldots, \\
& {[b(p-2)+1, b(p-1)-1], b(p-1) } \\
& {[b(p-1)+1, N-1]\} }
\end{aligned}
$$

where intervals $[i, j]$ having $i>j$ are removed from $\mathcal{I}^{\mathcal{F}_{i}^{N}}$.。 In essence, (34) is an RP sequence set that satisfies the control sequence property established in Theorem 3.

Having identified an RP that provides an admissible control sequence for all states of a particular facet, the following theorem shows that this same technique can be used to determine an RP sequence for any state in $\mathcal{R}^{N}$.

Theorem 4 ( $R P$ for Any Initial State): Given a state $x \in$ $\mathcal{R}^{N}$, let $\mathcal{F}_{(x)}^{N}$ be a facet through which a ray emanating from the origin through $x$ exits $\mathcal{R}^{N}$. Consider the RP sequence $\mathcal{I}^{R P}=\mathcal{I}^{\mathcal{F}_{(x)}^{N}}$ produced by Definition 14 for $\mathcal{F}_{(x)}^{N}$ and the associated RP $N$-step controllable set $\mathcal{R}^{R P}$ as per Definition 8. Then $x \in \mathcal{R}^{R P}$.

Proof: See [7, Theorem 6.1].

Theorem 4 , states that a valid RP is given by any RP polytope $\mathcal{R}^{R P}$ that includes the facet of $\mathcal{R}^{N}$ through which a ray from the origin in the direction of the current state $x_{k}$ exits. Therefore Definition 14 in combination with Definition 8 readily produce an RP set for any state $x_{k} \in \mathcal{R}^{N}$.

Having established a minimal RP sequence we can formulate a reduced parameterisation MPC optimisation problem of horizon $N$ for the current state $x_{k}=x$ as

$$
\begin{aligned}
& \mathcal{P}_{N}(x): \quad V_{N}^{O P T}(x) \triangleq \min V(\mathcal{X}, \mathcal{U}, N), \\
& \text { subject to: } \\
& x_{i+1}=A x_{i}+B u_{i} \text { for } i=0, \ldots, N-1, x_{0}=x, \\
& u_{j}=u_{l} \forall j, l \in \mathcal{I}_{i}^{\mathcal{F}_{(x)}^{N}} \text { for } i=0, \ldots,\left|\mathcal{I}^{\mathcal{F}_{(x)}^{N}}\right|-1, \\
& u_{i} \in \mathbb{U} \text { for } i=0, \ldots, N-1, \\
& x_{i} \in \mathbb{X} \text { for } i=0, \ldots, N, x_{N} \in \mathbb{X}_{f} \subset \mathbb{X},
\end{aligned}
$$

where $\mathbb{U}$ is as in (3), $\mathbb{X}=\mathcal{R}^{N} \times \mathcal{R}_{s}^{\max }$ is the feasible set, $\mathbb{X}_{f}=\left\{x_{N} \in \mathbb{R}^{n}: x_{N \mid u}=0\right\}$ is the terminal set, $V(\mathcal{X}, \mathcal{U}, N)$, where $\mathcal{X}=\left\{x_{0}, \ldots, x_{N}\right\}$ and $\mathcal{U}=$ $\left\{u_{0}, \ldots, u_{N-1}\right\}$, is the optimisation cost function and $\mathcal{I}^{\mathcal{F}_{(x)}^{N}}$ is the RP sequence as per Definition 14, constructed for the facet $\mathcal{F}_{(x)}^{N}$ through which a ray emanating from the origin through $x$ exits $\mathcal{R}^{N}$ (c.f. Theorem 4). Note that each set of indices $\mathcal{I}_{i}^{\mathcal{F}_{(x)}^{N}}$, for $i=0, \ldots,\left|\mathcal{I}^{\mathcal{F}_{(x)}^{N}}\right|-1$ introduces a sequence of equality constraints to the optimisation problem.

Note that the complexity of the above optimisation problem is directly proportional to the number of RP parameters which, as shown in Corollary 1, is less than or equal to $2 p-1$ (where $p$ is the number of unstable modes), and hence independent of the optimisation horizon $N$.

The above elements can be performed online at a relatively low computational expense. This approach has been used to implement a new variant of MPC called RP MPC whose details can be found in [8] for the second order case and in [7] for the more general case.

\section{CONCLUSIONS}

In this paper we analysed a class of input constrained $n^{\text {th }}$ order systems with real unstable poles and their associated $N$-step controllable sets.

With a view to reducing the number of control moves we introduced the concept of RP sequences which led to the development of RP $N$-step controllable sets. We identified the following significant properties in relation to these sets: (i) states originating on facets have associated control sequences that consist of a combination of groups of equal saturation control moves interspersed with free control moves. Further, the number of free moves has a relatively small upper bound determined by the number of unstable poles of the system. Hence, an equivalent control action can be achieved with RP sequences having fewer control moves of longer duration; (ii) For any state $x$ in an $N$-step controllable set, $\mathcal{R}^{N}$, an RP sequence is given by any RP polytope that includes the facet of $\mathcal{R}^{N}$ through which a ray from the origin in the direction of the state $x$ exits $\mathcal{R}^{N}$.

Finally, we have shown how RP sequences and sets can be used to implement MPC with a reduced number of parameters.

\section{REFERENCES}

[1] P.-O. Gutman and M. Cwikel, "Admissible sets and feedback control for discrete-time linear dynamical systems with bounded controls and states," IEEE Transactions on Automatic Control, vol. AC-31, no. 4, pp. 373-376, April 1986.

[2] J. B. Lasserre, "Reachable, controllable sets and stabilizing control of constrained linear systems," Automatica, vol. 29, pp. 531-536, 1993.

[3] T. Hu, D. E. Miller, and L. Qiu, "Null controllable region of LTI discrete-time systems with input saturation," Automatica, vol. 38, pp. 2009-2013, 2002.

[4] K. Muske and J. Rawlings, "Model predictive control with linear models," AIChe Journal, vol. 39, no. 2, pp. 262-2872, 1993.

[5] R. Cagienard, P. Grieder, E. C. Kerrigan, and M. Morari, "Move blocking strategies in receding horizon control," in 43rd IEEE Conference on Decision and Control, 2004.

[6] R. Webster, Convexity. Oxford University Press, 1994.

[7] A. Medioli, "Maximal controllability via reduced complexity model predictive control," Ph.D. dissertation, University of Newcastle. FEBE, School of Electrical Engineering and Computer Science, 2008. [Online]. Available: http://hdl.handle.net/1959.13/30688

[8] A. Medioli, M. Seron, and R. Middleton, "Reduced parameterisation MPC for input-constrained unstable linear systems Part 1: Implementation," in Proc. European Control Conference 2009 - ECC'09, Budapest, August 2009. 